\title{
Análisis de la vegetación, acuática y terrestre del complejo cenagoso La Grande de Beté, municipio Medio Atrato, Chocó, Colombia
}

\section{Analysis of the vegetation, aquatic and terrestrial of the complex La Grande de Beté, municipality Medio Atrato, Chocó, Colombia}

\author{
Giovanny Ramirez ${ }^{1}$, Zulmary Valoyes ${ }^{2}$
}

\section{RESUMEN}

El análisis de la vegetación asociada con el complejo de humedales la Grande de Beté, en la región del Medio Atrato, se realizó con el objetivo de determinar la estructura y composición vegetal. Para su desarrollo se aplicó una metodología que incluyó talleres con personas locales, complementado con muestreos de campo en cuatro unidades muestrales: rivera inundable, caño, espejo de agua y zona insular. Los resultados del estudio muestran la presencia de 239 especies distribuidas en 186 géneros y 67 familias; las angiospermas estuvieron representadas por 219 especies, agrupadas en 154 géneros y 53 familias, mientras los pteridofitos estuvieron representados por 12 familias, 16 géneros y 22 especies. Las familias mejor representadas corresponden a Leguminoceae con 28 especies, Arecaceae con 15 especies, Araceae con 12 especies, seguida de Bromeliaceae con 11 especies y Orchidaceae con 10 especies, las cuales se encuentran distribuidas en los diferentes microambientes asociados con este ecosistema. Se concluye que el estado de conservación de la ciénaga en general es bueno, lo que puede deberse a que no es una ciénaga aislada, forma un complejo de ciénagas y por lo tanto estas sirven como amortiguadoras retenedoras de desechos y otros agentes contaminantes que provienen directamente del río.

Palabras clave: Ciénaga; Estructura vegetal; Composición vegetal; Muestreo de campo.

\section{ABSTRACT}

The analysis of the vegetation associated to the complex of moist soils the Grande de Beté, in the region of the Atrato Means it was realized with the aim of determining the structure and vegetal composition. For its development a methodology that included meeting with local people, complemented with samplings of field in several units was applied samples: easilyflooded creek, water sewer and insular zone. The results of the study show to the presence of 239 species distributed in 186 sorts and 67 families; the angiospermaes were represented by 219 species, grouped in 154 sorts and 53 families, while the pteridofitos were represented by 12 families, 16 sorts and 22 species. The families better represented correspond to Leguminoceae with 28 species, Arecaceae with 15 species, Araceae with 12 species, followed of Bromeliaceae with 11 species and Orchidaceae with 10 species, which are distributed in different associated microatmospheres from this ecosystem. State of conservation of the bog generally is good, can be due to that it is not an isolated moist soils, forms a moist soils complex and therefore these serve like retaining shock absorbers as remainders and other polluting agents who come directly from the river.

(C) Instituto de Investigaciones Ambientales del Pacífico «John Von Neumann»
1. Biólogo, Magíster en Ciencias Biológicas, Investigador principal Componente Ecosistémico, Instituto de Investigaciones Ambientales del Pacífico (IIAP). e-mail: gramirezm3@hotmail.com

2. Bióloga, Especialista en Administración de Recursos Naturales, Docente Universidad Tecnológica del Chocó, Quibdó, Chocó. e-mail: zulmaryvaloyes@hotmail.es Recibido: enero 14, 2009 Aceptado: enero 28, 2009 


\section{Vegetación acuática y terrestre del complejo La Grande de Beté. G. Ramírez, Z. Valoyes}

Keywords: Moist soils; Vegetal structure; Vegetal composition; Sampling of field.

\section{INTRODUCCIÓN}

El Atrato es el tercer río más importante en el país después del Amazonas y el Magdalena, por el gran aporte hídrico que estos y sus afluentes realizan, convirtiendo a Colombia en uno de los cuatro países con mayor disponibilidad de recurso hídrico del planeta (Castellanos 2006). A pesar de lo anterior, en Colombia no se tiene un dato preciso del número de humedales. Se han adelantado estrategias para su conocimiento y conservación, por la importancia que representan estos ecosistemas. En este contexto se han identificado 27 complejos de humedales, $30 \%$ presentes en el Caribe y $70 \%$ en el Magdalena medio y en el río Atrato (Instituto Alexander Von Humboldt 1998).

En el departamento del Chocó se han realizado algunos estudios en estos ecosistemas, sin embargo, la información acerca de la composición florística de estos es incipiente; la mayoría de estudios se enfocan principalmente en el componente faunístico, no obstante se han realizado algunos inventarios y planes de manejo en áreas cercanas, donde se mencionan datos acerca de la flora asociada con este tipo de ecosistemas entre estos tenemos los de Salazar (2000), Ramos (2002), Rangel (2004), Cárdenas (2006) y CORPOURABA \& CODECHOCO (2006). Estos sistemas cenagosos se han descrito como un potencial de desarrollo para las comunidades asentadas en su zona de influencia, las cuales pueden definirse por las características ecológicas permisibles de ser analizadas por medio de observaciones holísticas desde algunas de las particularidades que tienen determinados sistemas, sobre todo los que convergen en el área de influencia del Pacífico colombiano, porque, presentan condiciones especiales, como una alta precipitación, altas temperaturas del ambiente, bosque circundante diverso que le permite adquirir mayor contribución de materiales que proporcionan los diferentes nutrientes que sostienen la estructura y función de estos sistemas.

\section{ÁREA DE ESTUDIO}

Las evaluaciones ecológicas rápidas se llevaron a cabo en cuatro áreas correspondiente a la ciénaga La Grande; las zonas de muestreo están ubicadas dentro y fuera de la ciénaga; se escogieron cuatro sitios representados por diferentes microambientes cada uno determinados por unidades paisajísticas similares, los cuales se ubican espacialmente de la siguiente manera:

a. Llanura aluvial.Ubicada en la margen izquierda del río Atrato en sentido sur-norte, dentro de las coordenadas planas $1035893 \mathrm{Y}$; $1157869 \mathrm{X}$, a una altura de $40 \mathrm{msnm}$ aproxima- damente; se incluye dentro del complejo de humedales de la comunidad de Beté.

b. Caño «Torrobon». Ubicado en la margen izquierda del río Atrato en sentido sur-norte, dentro de las coordenadas planas $1035083 \mathrm{Y}$; $1157757 \mathrm{X}$, a una altura de $41 \mathrm{msnm}$ aproximadamente.

c. Espejo de agua (CG). Ubicada en medio del complejo cenagoso La Grande, dentro de las coordenadas planas $1034646 \mathrm{Y}$; $1156803 \mathrm{X}$, a una altura entre 40-45 msnm aproximadamente;

d. Zona insular. Ubicada dentro del complejo cenagoso La Grande en los alrededores de la ciénaga grande, dentro de las coordenadas geográficas de $6^{\circ} 00^{\prime} 59.3^{\prime \prime} \mathrm{N} ; 76^{\circ} 46^{\prime} 44.7^{\prime \prime} \mathrm{W}$, a una altura entre 54-59 msnm aproximadamente.

\section{METODOLOGÍA}

Se realizaron talleres con los habitantes del municipio, principalmente con aserradores y conocedores de plantas, donde dieron a conocer los nombres vulgares de las principales especies presentes y aprovechadas por los habitantes de la región, información que fue corroborada en campo a través observaciones y muestreos al azar en la zona de estudio, con el objetivo de analizar la vegetación a partir de los atributos de riqueza y estructura florística; se colectaron individuos de plantas vasculares presentes en cada una de las unidades muestreales, de los cuales se seleccionó material preferiblemente fértil para su posterior identificación; se observó y anotó el hábito de crecimiento de cada una de las especies y se tuvo en cuenta el diámetro a la altura del pecho (DAP) $>10$, además, se tomaron datos de altura y nombre vulgar. Debido a la complejidad, amplitud y la época de inundación del área no se realizó un muestreo sistematizado; sin embargo se diferenciaron cuatro ambientes (rivera o planicie inundable, caño, espejo de agua y zona insular), donde se realizaron muestreos aleatorios tratando de recopilar la mayor información posible sobre vegetación. El material colectado se prensó, etiquetó y secó. Este se identifico con la ayuda de claves taxonómicas y luego se depositó en el herbario de la Universidad Tecnológica del Chocó «Diego Luis Córdoba», Quibdó.

\section{RESULTADOS Y DISCUSIÓN}

Las observaciones realizadas en la totalidad del ecosistema permitieron tener visión amplia de la zona y hacer una división de los tipos de ambiente presentes en el área de estudio. Vale la pena resaltar que el muestreo coincidió con la época de inundación lo que facilitó el acceso al dosel de algunos ambientes.

Los resultados del estudio florístico muestran la presencia de 239 especies distribuidas en 186 géneros y 67 familias. 
Bioetnia Volumen 6 No 1 (enero-junio), 2009

Tabla 1

Especies en diferentes categorías de amenaza

\begin{tabular}{lll}
\hline \multicolumn{1}{c}{ Especie } & Familia & Categoría de amenaza \\
\hline Mauritiella macroclada (Quitasol) & Arecaceae & \\
Anacardium excelsum (Caracolí) & Anacardiaceae & Casi amenazado \\
Campnosperma panamense (Sajo) & Anacardiaceae & Casi amenazado \\
Huberodendron patinoi (Carrá) & Bombacaceae & Vulnerable \\
Prioria copaifera (Cativo) & Caesalpinaceae & En peligro \\
Orphanodendrum bernalii (Tirateté) & Caesalpinaceae & Vulnerable \\
Dipteris leifera (Choibá) & Fagaceae & Casi amenazado \\
Quercus bumboldtii (Roble) & Fagaceae & Vulnerable \\
Humiriastrum procerum (Chanul) & Humiriaceae & Peligro crítico \\
Cariniana pyriformis (Abarco) & Lecythidaceae & Peligro crítico \\
Cedrela odorata (Cedro) & Meliaceae & En peligro \\
\hline
\end{tabular}

Las angiospermas estuvieron representadas por 219 especies, agrupadas en 154 géneros y 53 familias, mientras los pteridofitos estuvieron representados por 12 familias, 16 géneros y 22 especies.

Las familias mejor representadas corresponden a Leguminoceae (Fabaceae Caesalpinaceae, Mimisaceae) con 28 especies, Arecaceae con 15 especies, Araceae con 12 especies, seguida de Bromeliaceae con 11 especies y Orchidaceae con 10 especies, las cuales se encuentran distribuidas en los diferentes microambientes asociados con este ecosistema. Es de resaltar que la distribución vertical de las especies permitió diferenciar los siguientes hábitos: herbáceo, arbustivo, arbóreo, epifito, hemiepifito, dominando los tipos de ambientes seleccionados, rivera del río (arboles y pastizales); caño (arbustos, arboles, epifitas, hemiepifita); espejo de agua (vegetación flotante, árboles, epifitas, vegetación enraizada); región insular (arbusto, árboles, epífitas, hemiepifitas).

De las 219 especies de angiospermas que ocurren en la Ciénaga Grande, 11 se encuentran con alguna categoría de amenaza, según Cárdenas \& Salinas (2007) 10 de estas especies se encuentran listada en el libro rojo de especies forestales (Tabla 1); Rangel (2004) incluye a Mauritiella macroclada como especie amenazada.

Aunque el estudio se realizó sobre el espejo de agua, para un mejor entendimiento del esqueleto vegetal y la dinámica trófica de este ecosistema se tuvieron en cuenta cuatro microambientes. El análisis de estos permitió tener una visión integrada de las características morfoestructurales del ecosistema en conjunto para poder definirlos y diferenciarlos teniendo en cuenta patrones de estructura, de diversidad, de dinámica y del aporte que cada uno de estos ambientes hace a la ciénaga.
Microambiente 1 (rivera del río o planicie inundable). Este microambiente se encuentra directamente influido por la dinámica hídrica del río Atrato, característica que le permiten mantener grandes descargas de materia orgánica en períodos de inundación, convirtiéndose en la zona más productiva, razón por la cual en ésta es donde se establecen cultivos agrícolas (plátano, chontaduro, árbol del pan, caña, arroz y especies forestales manejadas como cedro).

A nivel florístico este microambiente se caracteriza por la presencia de bosques siempre verde donde se evidencia la degradación de masas boscosas; la vegetación se encuentra distribuida a lo largo de la llanura aluvial, donde se presentan suelos bien drenados y la vegetación es dominada por pastizales de Leersia hexandra, Paspalum repens e Hymenachne amplexicaulis, Hydrocotyle cf. Umbellata, Jussiaea natans, Neptunia oleoraceay Utriculariafoliosa; herbazales de Polygonum acuminatum, Aechmea magdalenae, Axonopus compressus, Pennisetum purpureum, Hydrocotyle bonplandii,Ludwigia affinis, Thalia geniculata (Rangel 2004) acompañados por Calathea lutea, Dimerocostus strobilaceus y Scleria malaleuca. Está zona incluye unidades de paisajes de bosque ribereño que presenta elementos florísticos dominados por Ficus spp., Inga edulis, Inga sp., Cedrela odorata, Artocarpus artilis, Euterpe oleraceae, Apeiba aspera, Vismia latisepala, Cecropia insignis, Cecropia spp., Vismia latisepala. En el río Amé afluente del Atrato, es común observar relictos de Prioria copaifera, así como la presencia de naidizales (Euterpe oleoracea) asociados conBactris bongiartii y Cecropiasp. A pesar de lo anterior en esta zona se observó menor diversidad florística lo cual se puede deber a la implementación de cultivos en la margen del río (Figura 1). Observaciones reali- 

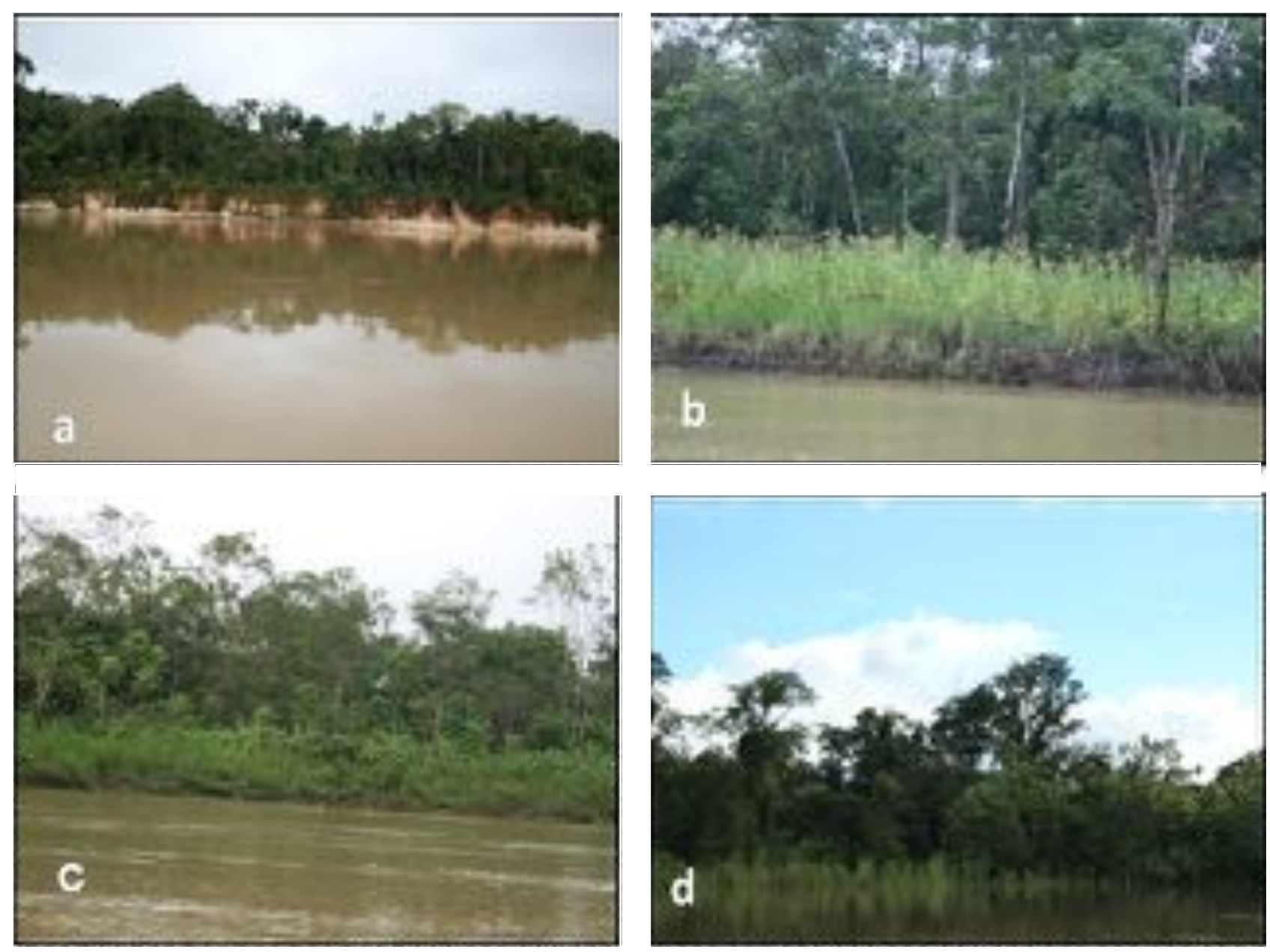

Figura 1. Vegetación ribereña. a. Ribera del río erosionada; b. Cultivos de plátano y cedro;

c. Vegetación dominada por pastizal en asociación con cedro; d. Vegetación dominada por poáceas y cecrópiaceas.

zadas en este sitio revelan la alta diversidad faunística e interacción con la vegetación, porque esta zona se constituye en fuente de alimentación para mamíferos (primates, pericos) y algunas aves (rapaces, playeras y pescadores).

Microambiente 2. Caño. Este microambiente se encuentra entre la zona de transición de las aguas sedimentadas y cargadas de materia orgánica del río Atrato y las aguas pardas de las ciénagas circundantes; la vegetación característica de este microambiente es propia de bosque de pantano; hacia la entrada del caño se puede observar una vegetación arbórea y arbustiva influida por la vegetación de rivera con asociaciones deMimosa (spp.),Inga (spp.), Clusia (spp.),Spathiphylum friedrichsthalii, Piper sp., Topobea sp., Souroubea guianensis, Drimonia serrulata, Sphaeradenia sp., Anthurium formosum, Anthurium obtusum, Monstera sp., Philodendron (spp.), Sanchezia macrocnemis, Costus scaber, Souraroubea guianensis, Erythrina costaricencis vr. Panamensis, Heliconia latispata, Anemopaegma chamberlaynii, Clytostomabinatum, Calatea clotalifera, Pereromia montium, Clusia minor, Palicourea guianensis, Heisteria acuminata.

Hacia la parte media del caño se encuentran remanentes de Prioria copaifera, sus frutos se convierten en fuente de alimento sobre todo de primates (Piedra \& Bravo 2002), se encuentran acompañados por comunidades de Inga sp., Pachira acuatica; en la parte que conecta el caño con la ciénaga hay una vegetación constituida por comunidades de Bactris brongniartii, arbustos de Ludwigia palustris, L. inclinata y varias especies de leguminosas. En esta área la diversidad es mayor con respecto a la rivera del río, el estrato herbáceo es nulo debido a que la vegetación de este microambiente se encuentra sometida a largos períodos de inundación que sólo permite el desarrollo del estrato arbustivo y arbóreo, con una alta frecuencia de lianas y epifitas que 

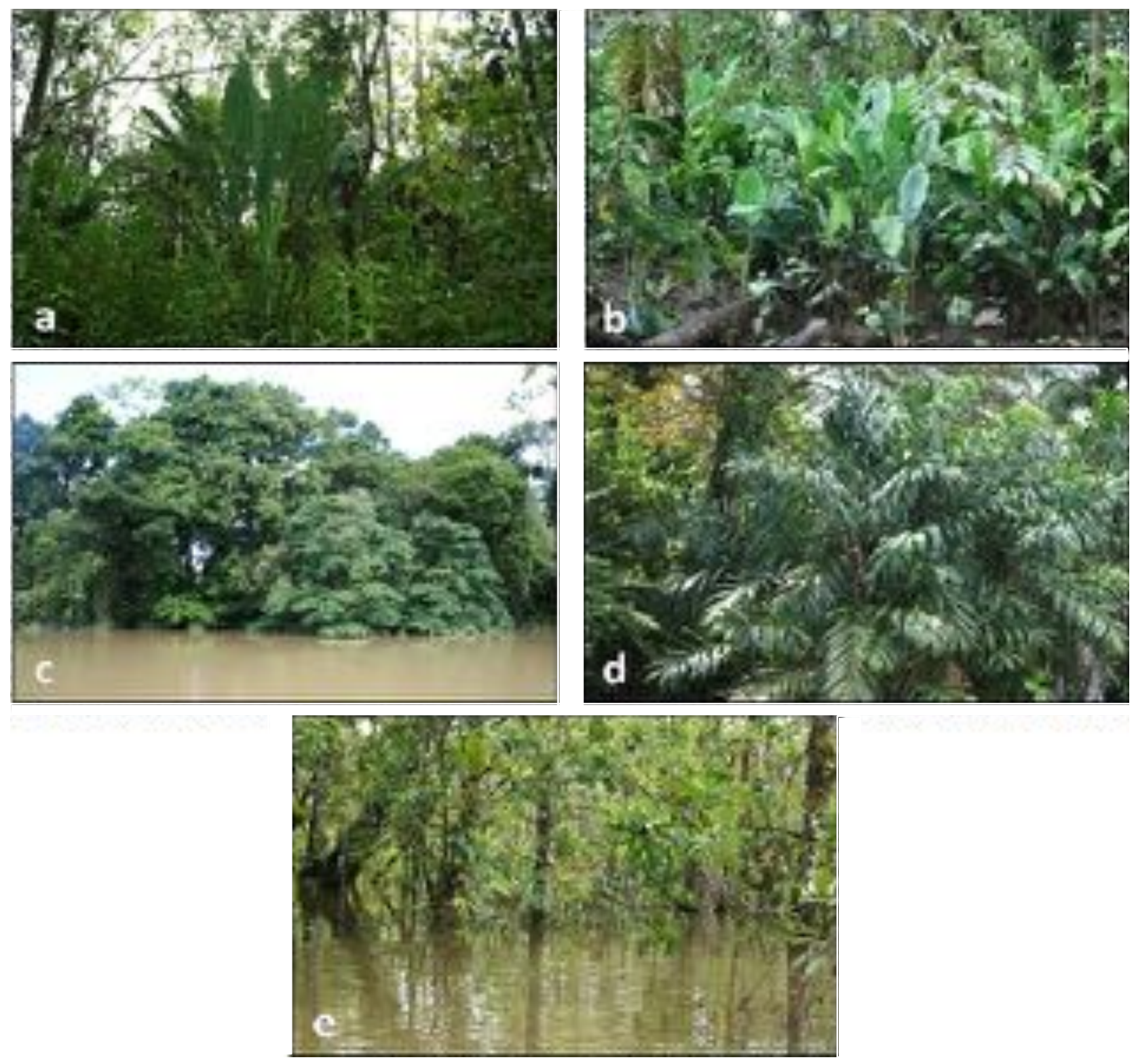

Figura 2. Panorama de la vegetación del Caño. a. Asociaciones de Cyclanthus sp., Heliconia sp., Costus spp., Cecropia sp.; b. Spathiphylum friedrichsthalii; c. Remanentes de Prioria copaifera; d. Comunidad de Bactris brongniarti; e. Vegetación al final del caño.

hacen que el esqueleto vegetal sea más complejo (Figura 2). La presencia de este tipo de vegetación convierten este ambiente en la zona ideal para el establecimiento de hábitat de una fauna variada que incluye mamíferos (primates y roedores), aves de sotobosque, anfibios y reptiles. Este caño se convierte en el paso obligado de esta fauna hacia el humedal incluyendo la ictiofauna residente que realiza migraciones para reproducirse y/o alimentarse en el cauce del río.

Microambiente 3. Espejo de agua (zona limnetica, z. litoral y z. riberina o marginal). Esta ciénaga es la más grande del complejo que incluye las ciénagas de Torrobón, Lava pan, La Grande y Bosque Largo. Las dos primeras reciben descargas de agua, que incluye materia orgánica, agentes contaminantes y residuos sólidos provenientes del río. Lo anterior hace que actualmente se encuentren con un cierto grado de deterioro; estas actúan como filtro de la ciénaga La Grande convirtiéndola en un humedal bien conservado.

Zona limnetica. Ubicado en el centro del humedal con una gran extensión de fondo que supera el límite fótico, se encuentra desprovista de macrófitas, la gran cantidad de 

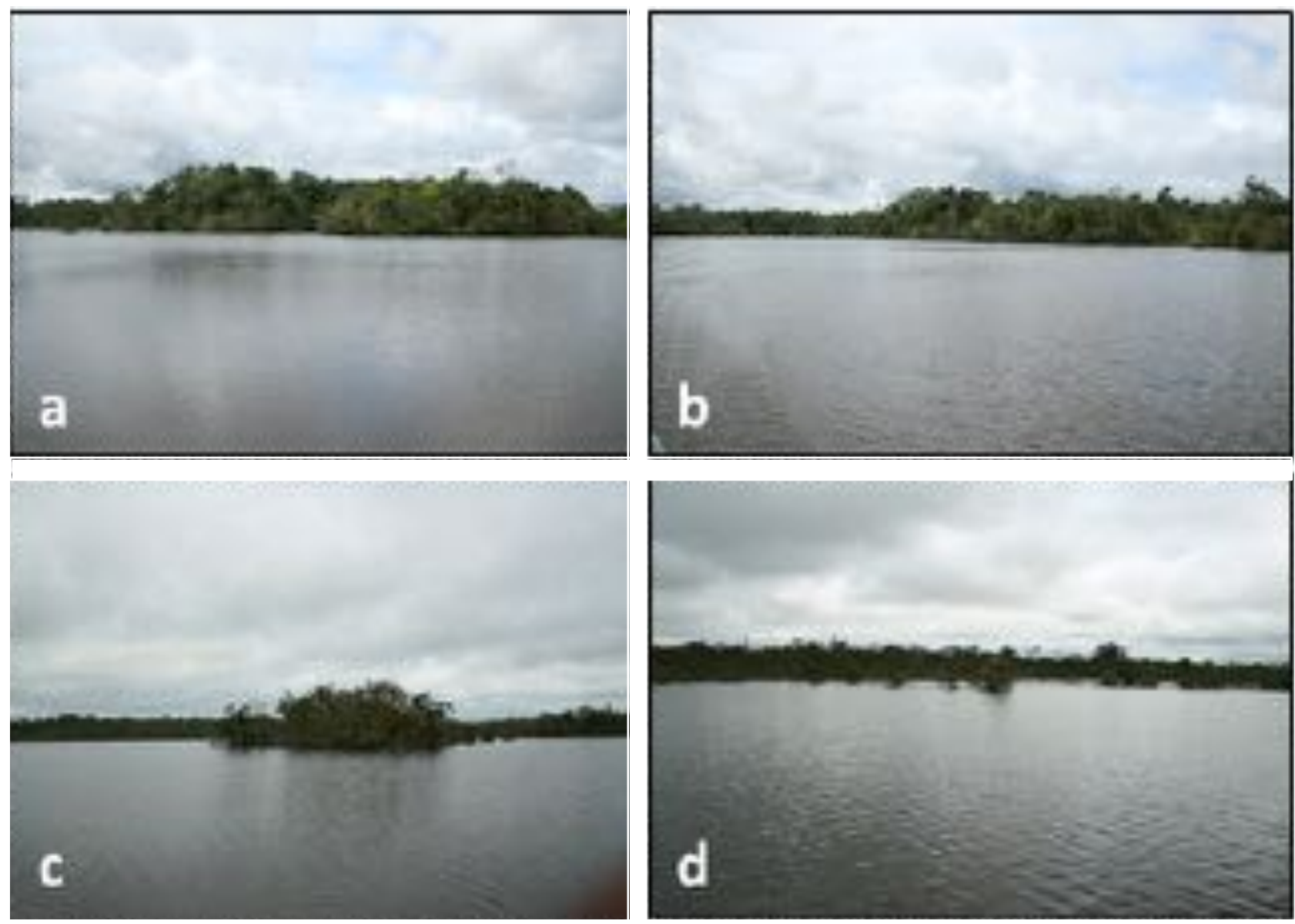

Figura 3. Zona limnetica a, b, c y d. diferentes ángulos de área desprovista de macrófitas.

materia orgánica procedente de las asociaciones vegetales que residen en las zonas aledañas le dan una coloración que varía entre pardo oscuro a negruzca (Figura 3).

Zona litoral. Comprende desde el contacto de la zona fótica del humedal, hasta el inicio de la formación de macrófitas emergentes o enraizadas al sedimento. Se caracteriza por presentar islas flotantes que forman grandes asociaciones con especies dominantes como Ludwigia sedioides, Azolla microphylla, Nymphoides indica, Eichornia craspes, Cyperus luzulae, Ceratophyllum demersum (Figura 4). Esta última se encuentra como único elemento de la vegetación acuática, lo que posiblemente no permite la presencia de la fauna aviar, tal vez por la poca oferta de alimento que provee; mientras las macrófitas arriba mencionadas, pueden encontrarse solas o mezcladas convirtiéndose en el sustrato de algas ideal para la alimentación de peces herbívoros residente en la ciénaga, así como el hábitat propicio de una gran diversidad de macro invertebrados que se constituyen en la dieta alimenticia de una variedad de aves acuáticas (chorlos, curvo patos, etc.).

Estos tapetes de macrófitas se encontran enraizadas y sumergidas o flotando sobre la superficie del agua, situación que se puede convertir en un problema para los pobladores locales porque su condición de flotantes les permiten desplazarse de un lado a otro de la ciénaga taponando la vía de acceso e impidiendo la entrada o salida del mismo (Figura 5). El período de vida corto de estas plantas les permite permanecer en constante renovación generando abundancia de biomasa y materia orgánica que al descomponerse pueden convertirse en la causa principal de eutroficación del humedal, no obstante cumplen un papel ecológico muy importante al convertirse en productores primarios de las cadenas tróficas produciendo gran cantidad de oxígeno en el ecosistema.

Zona riberina o marginal. Comprende el contorno de la ciénaga, asentada en un sustrato edáfico mal drenado, permanentemente inundado; la vegetación de esta zona se caracteriza por ser hidrófita y anfibia adaptada a vivir con exceso de agua. Es frecuente observar comunidades de Bactris brongniartii y algunos individuos de Montrichardia arborescens. La vegetación arbórea es escasa, sin embargo, se encuentra dominada por Pachira acuatica y algunas especies de leguminosas (Inga spp.), que soportan una gran 


\section{Bioetnia Volumen 6 No 1 (enero-junio), 2009}

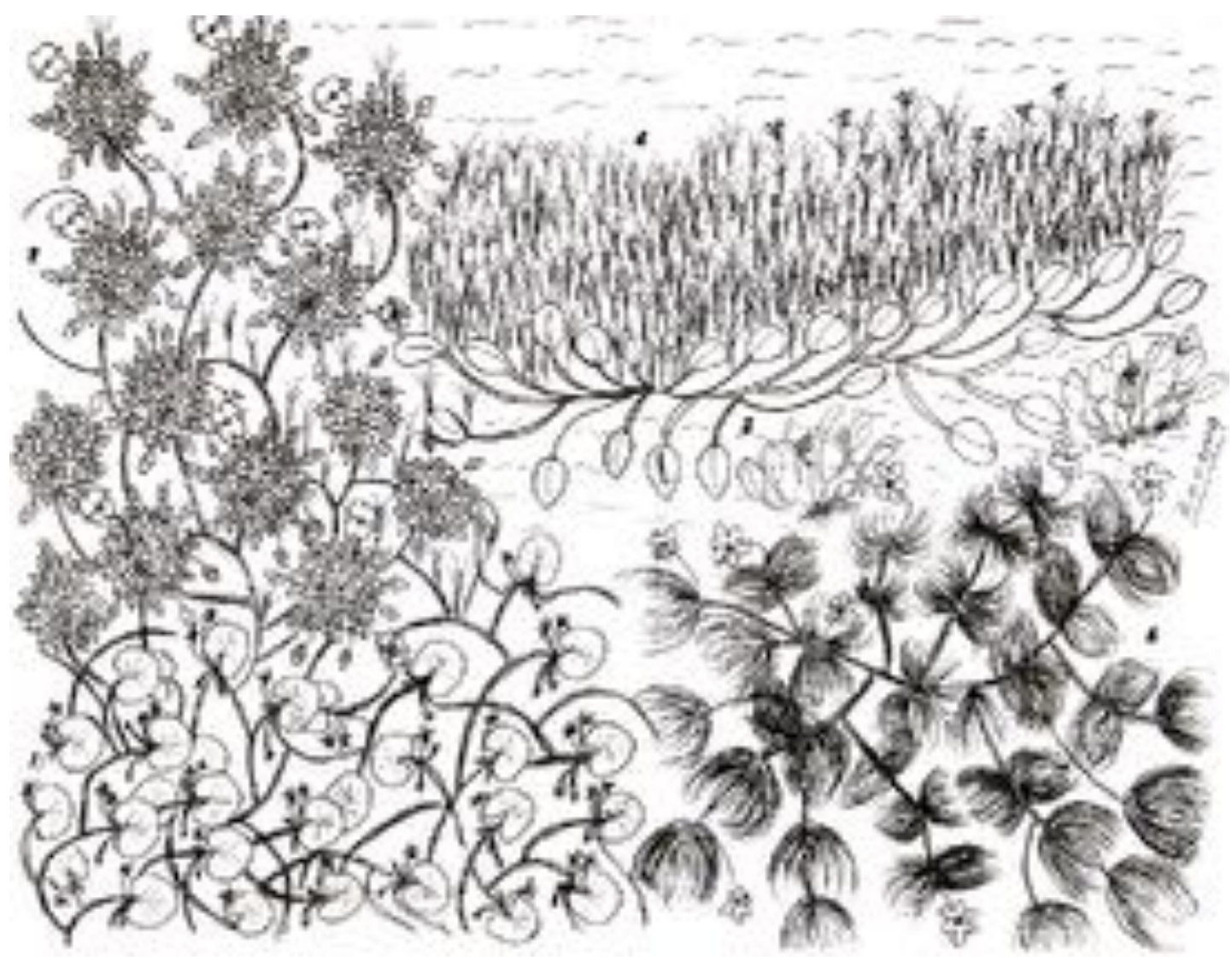

Figura 4. Panorámica de la vegetación enraizada y flotante de la zona litoral. 1. Nymphoides indica; 2. Ludwigia sedioides; 3. Eichornia craspes; 4. Cyperus luzulae; 5. Ceratophyllum demersum.

cantidad epifitas (Figura 6).

Las epifitas en esta zona se encuentran dominadas principalmente por las orquídeas (Erycina sp., Dimerandra latipetala, Ocidium sp., Sobralia sp., Trigonium argentinodium, Epidendrum nocturna, Maxilariasp., Rudolphiela picta), seguida por las bromelias (Werauhia sanguinolenta, Werauhia grandiflora). Con menor frecuencia se observaron especies de Anemopaegma chamberlaynii, Topobea parasitica, Cavendischia callista,Bonisteriopsis martiniana vr subenervia, Epiphyllum phyllanthus, Anthurios spp. (Figura 7). La dominancia de epifitas se debe a la facilidad que poseen estas de colonizar sobre todo ambientes húmedos y raparíos; en esta área es frecuente encontrar asociaciones de macrófitas con las especiesLudwigia sedioides,Nymphoides indica, Eichornia craspes y Cyperus luzulae.

La ocurrencia y establecimiento de especies como Epiphyllum phyllanthus (Cactaceae), se debe posiblemente a que es una especie que habita en el interior de bosques húmedos tropicales (Betancur et al. 2007), sus frutos por ser carnosos podrían constituirse en la dieta de algunas aves que inciden en este tipo de ambientes.

Microambiente 4. Zona insular. Se caracteriza por pre- sentar un nivel freático alto, el terreno presenta una leve pendiente, en épocas de inundación el nivel del agua sube hasta cierta parte arrastrando una gran cantidad de materia orgánica en descomposición (hojarasca) que alberga una variada artropofuana, la cual sirven de alimento para anfibios y reptiles. En épocas de verano estas islas deben estar conectadas lo que explica la presencia de pequeños roedores. A nivel florístico se caracteriza por la presencia de relictos de bosque primario con estrato herbáceo ralo, posiblemente por la densidad del dosel que no permite la penetración directa de los rayos del sol permitiendo el desarrollo de semillas de plantas residentes que aprovechan los destellos de luz para el crecimiento de sus plántulas, afectando el establecimiento de especies halófitas- Sin embargo, se observan plantas de porte herbáceo de hasta 1 metro de altura donde predominan Monotagma laxon, Maclenia smithiana, Displasia karatifolia, Fimbristilis annua, Mapania sylvatica (Figura 8), incluyendo una amplia gama de helechos que encuentran en este ambiente húmedo y sombrío el sitio ideal para desarrollarse, distribuyéndose desde el suelo hasta el dosel. En el sotobosque ocurren algunas especies arbustivas de Tococa guianensis, Miconia sp., Camaedorea sp., Geonomatriandra, 

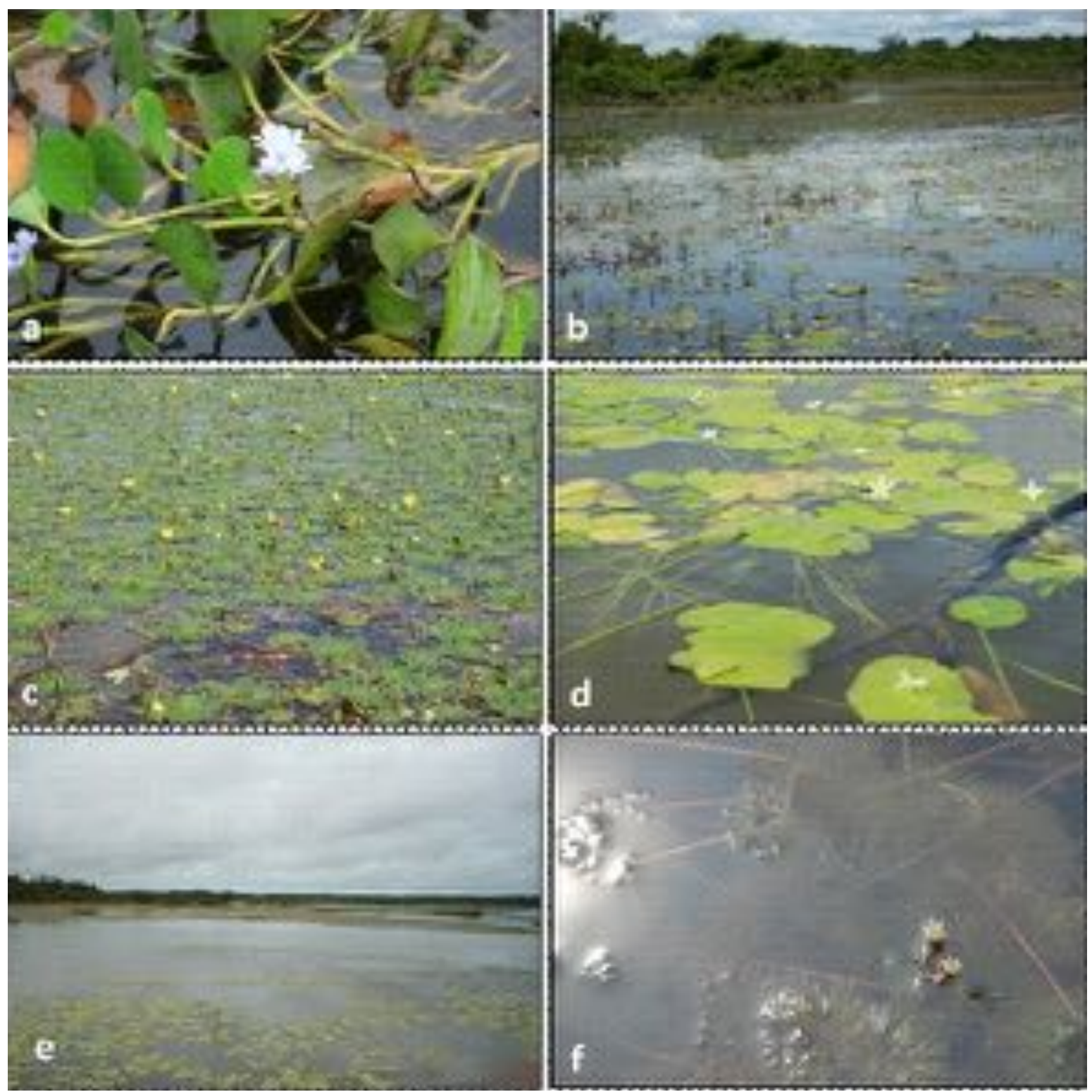

Figura 5. Macrófitas flotante. a. Eichornia crasipes; b. Vegetación enraizada y flotante (Ludwigia spp., Nymphoides indica); c. Ludwigia Ludwigia sedioides; d. Nymphoides indica; e. Vista de tapetes de macrófitas; f. Macrófita sumergida Ceratophyllum demersum.

Geonoma sp., Piper arborium. El estrato más predominante es el arbóreo con la frecuencia de especies como Sloanea zuluaensis, Vochysia jefensis, Huberodendron patinoi, Humiriastrum procerum, Eschueilera pitierii, Mabea occidentalis, Beilschmiedia rohliana, Calophyllum longifolium, Protium veneralense, Eugenia lineatifolia, Tapirira myrianthus, Matisia castano, Ocotea cernua, Guateria sp., Anibasp., Cespedecia spatulata, Ambaliasp., Pera arborea, Molovetia sp., Sclerolobium sp., Couma macrocarpa,Pouteria sp. La diversidad de especies arbóreas han permitido que este microambiente sea el sitio propicio para la colonización de mamíferos (primates, roedores) y se ha convertido en el hábitat de aves de sotobosque y aves rapaces que habitan este ecosistema.

La alta frecuencia de lianas y epifitas contribuyen a la complejidad de la estructura boscosa en este microambiente; las epifitas dentro del bosque se distribuyen desde niveles más bajos de lo normal hasta el dosel, patrón que de acuerdo con (Arevalo \& Betancur 2004) puede estar ligado con la humedad continua por la cercanía al humedal, fenómeno que 


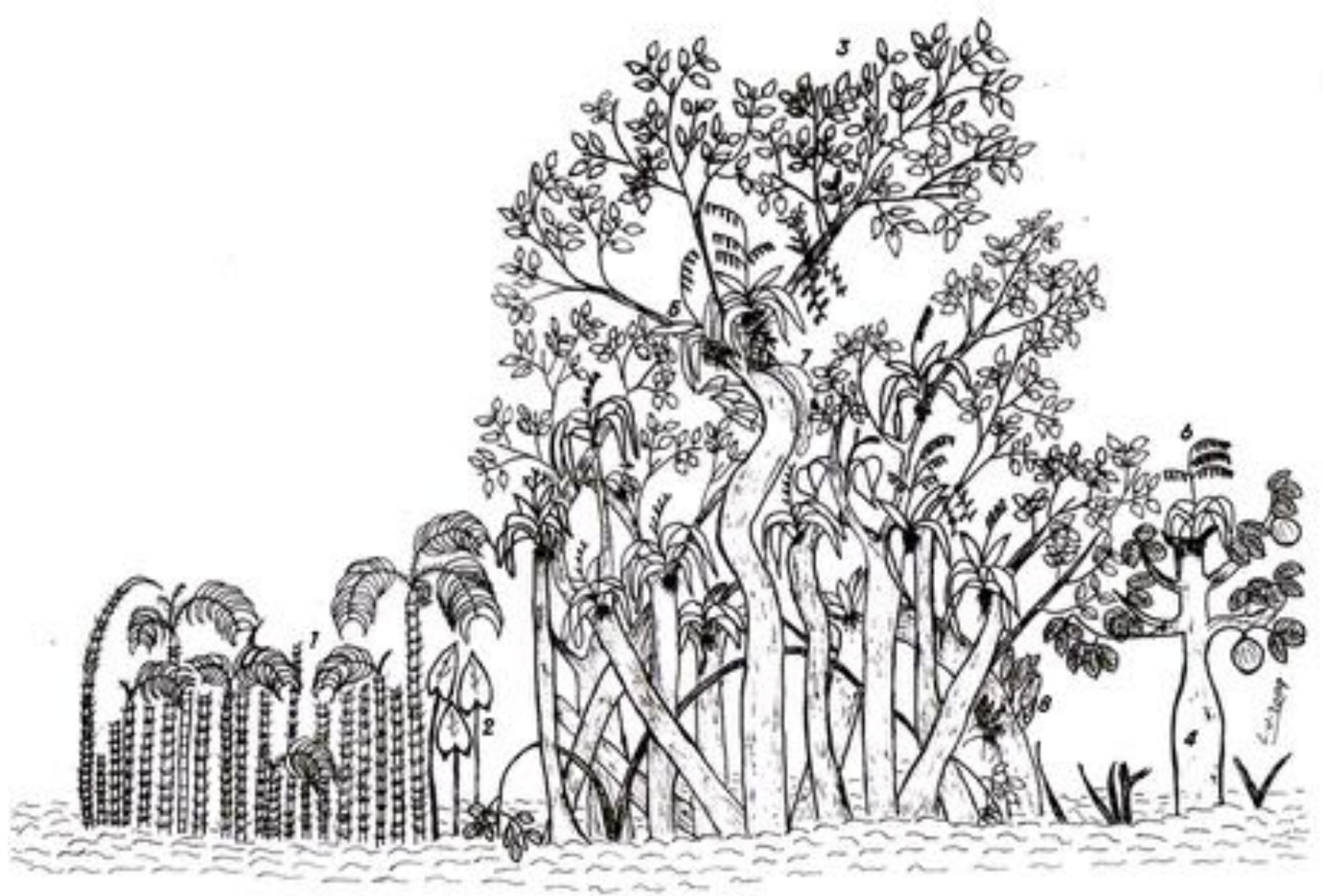

Figura 6. Perfil idealizado de la vegetación riberina o marginal. 1. Bactris brongniartii; 2. Montrichardia arborescens; 3. Inga spp.; 4. Pachira acuatica; 5. Werauhia grandiflora; 6. Werauhia sanguinolenta; 7. Epiphyllum phyllanthus; 8. Maxilaria sp.

podría estar facilitando la colonización de epifitas en todos los estratos del bosque en cualquier época del año. En este sentido las especies más representativas corresponden a Anthurium bakeri H., A. silvanii H., Philodendron fragantissimum H., P. heleniae T. B.,Dichaea sp., Guzmania lingualata, G. musaica, Tillandsia anceps, T. monadelpha, T. bulbosa, Dioscoria sp.

De acuerdo con las observaciones realizadas en cada una de estas islas se puede indicar que la isla $\mathrm{N}^{\mathrm{o}} 1$ (Figura 9) presenta una mínima intervención antrópica en relación con la $\mathrm{N}^{\mathrm{o}} 2$, en donde se evidencia una fuerte presión dejada por la entresaca de especies arbóreas de interés comercial, con respecto a la $\mathrm{N}^{\circ} 3$ se percibe una situación más lamentable; los árboles maderables de interés comercial, fueron arrasados en su mayoría; en esta isla se evidencia un estado sucesional temprano del bosque, las especies se encuentran en pleno crecimiento, hay una clara diferencia entre el estrato arbustivo y el arbóreo, la estructura es menos compleja que en las islas anteriores y la abundancia de los individuos es mayor disminuyendo notablemente la diversidad. Las especies de porte arbóreo no alcanzan un diámetro superior a los 20 cm situación que evidencia la explotación forestal reciente a la que fue sometida esta área del bosque por parte de los pobladores.

La riqueza florística encontrada en la ciénaga muestra patrones generalmente similares a los hallados en el complejo cenagoso los Platillos y las ciénagas de Tumarado, Perancho, la Honda y la Rica ubicadas en el delta del bajo Atrato (Duque et al. 2003, Ramos 2002) y a los reportados por Cárdenas (2006) para el Cerro del Cuchillo. Los estudios previos corroboran la apreciación de Rangel (2004), quien define la llanura aluvial del Atrato como una matriz continua desde el medio Atrato hasta su desembocadura; vale la pena resaltar que los atributos de diversidad tanto de riqueza como de abundancia no son comparables porque difieren en tiempo y metodología.

La estructura y composición florística del bosque del humedal es alta y contribuye a mejorar el conocimiento de la vegetación de la zona, porque existen vacios de información como los reportados por CORPOURABA y CODECHOCO (2006) en el plan de manejo de complejos de humedales del bajo Atrato; estos manifiestan que el componente florístico 

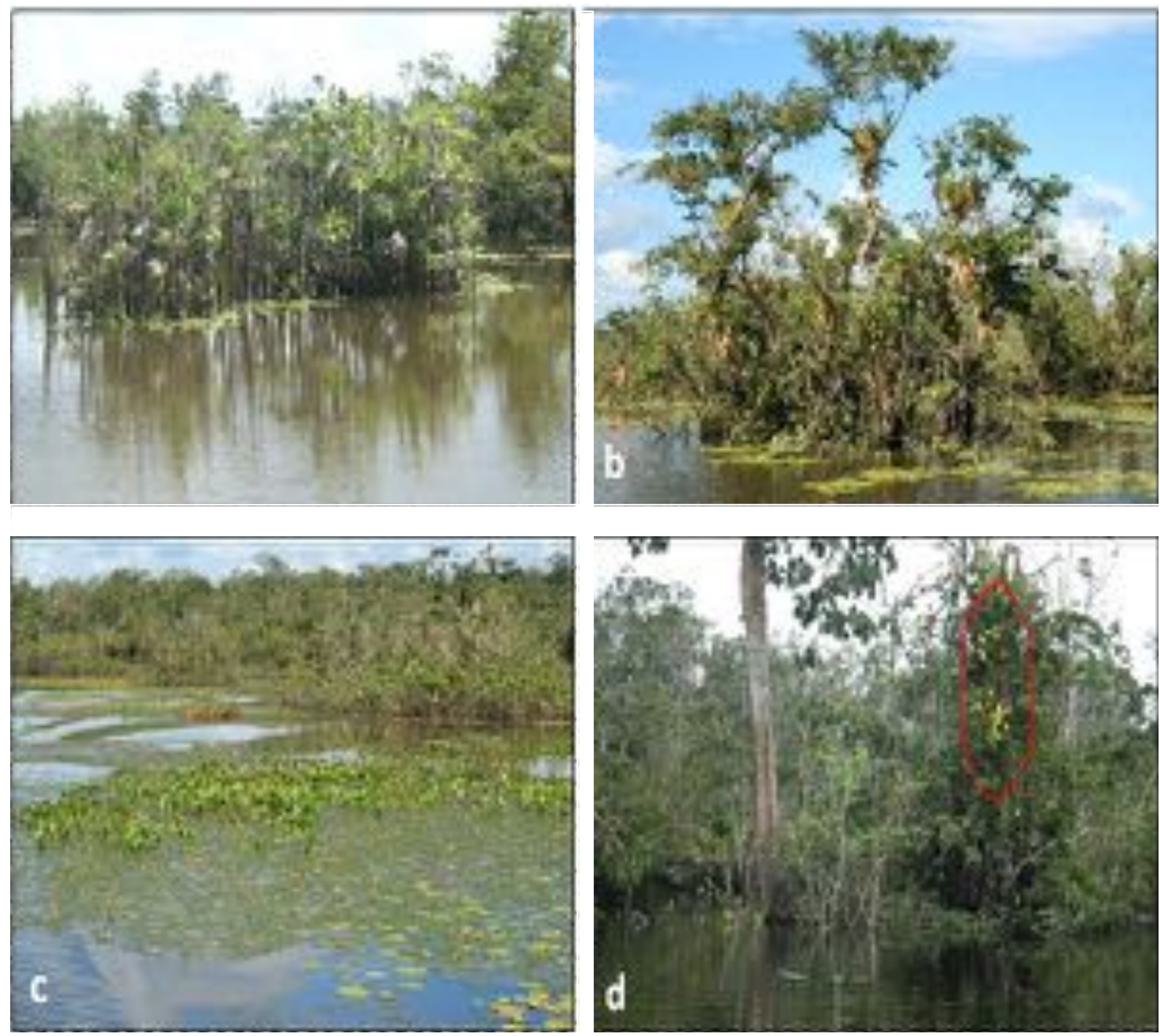

\section{Figura 7. Vegetación riberina. a. Comunidad de Bactris brongniartii; b. Árbol cargado de plantas epifitas; c. Macrófitas enraizadas y flotantes; d. Pachira acuatica, Árbol cargado de epifitas (Bonisteriopsis martiniana vr subenervia).}

para el bajo Atrato es casi inexistente debido a los incendios forestales que se presentaron en la década de 1990 y a las condiciones de anegabilidad del terreno en otras áreas.

La composición de especies en el espejo de agua muestran mayor riqueza y abundancia comparado con los estudios realizados por CORPOURABA y CODECHOCO (2006) y Ramos (2002); lo anterior permite mostrar la diversidad trófica para el área muestreada.

\section{CONCLUSIONES}

El estado de conservación de la ciénaga en su contexto general es bueno, lo que puede deberse a que no es una ciénaga aislada, forma un complejo de ciénagas y por lo tanto estas sirven como amortiguadoras retenedoras de desechos y otros agentes contaminantes que provienen directamente del río.

El dominio de poblaciones de Ceratophyllum demersum (macrófita sumergida a excepción de sus flores), encontradas como única especie en este sector (bosque largo) de la ciénaga se debe posiblemente a la competencia por sus altos requerimientos de nutrientes, lo cual no permite el establecimiento de otras macrófitas en esta área del humedal; además, la coloración del agua posiblemente muestre la secreción de algunas sustancias químicas procedentes de la especie que no permiten el establecimiento de otras macrófitas. 

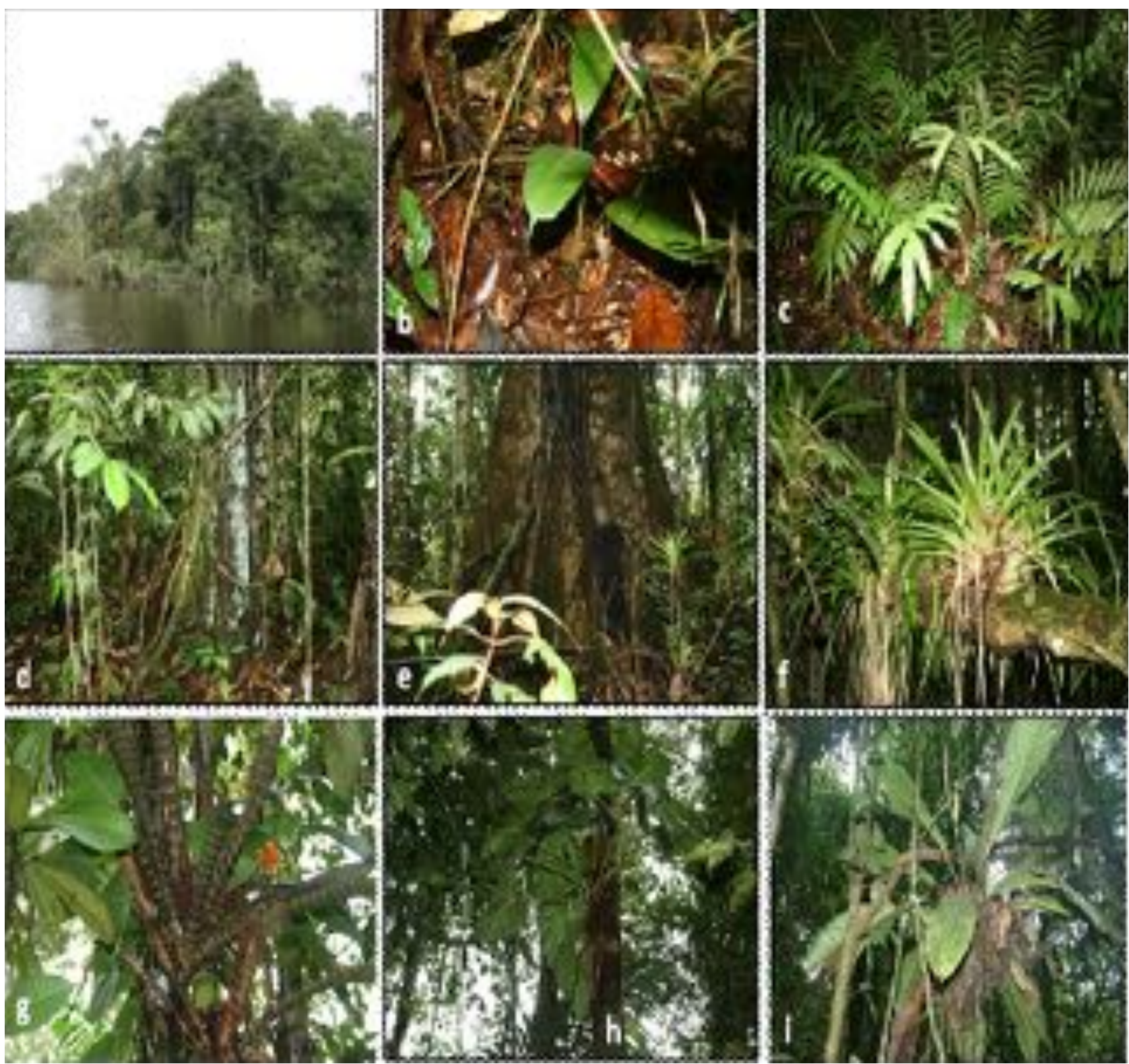

Figura 8. Región insular. a. Vista frontal de la isla 1; b. Sustrato cubierto por materia orgánica y plántula (Wettinia quinaria); c. Helechos presentes en la isla (Polypodium sp-); d. ILianas; e. Raíz de Huberodendrum patinoi; f, g, h. Plantas epifitas; i. Lianas y epifita.

Las fabáceas y las arecáceas son el grupo de plantas vasculares de porte arbóreo más importante en cuanto a riqueza y a abundancia dentro de la ciénaga, debido a la facilidad que poseen de adaptarse a las condiciones de humedad en este tipo de ecosistemas.

La presencia de macrófitas en esta ciénaga es un indicador de buen estado, porque estas se convierten en fuente de alimento y hábitat para una diversidad de fauna residente y estacional.
La explotación forestal por ser a baja escala y por realizarse de forma manual permite una rápida recuperación del bosque; a esto se suma las temporadas marcadas de la actividad pesquera que permite que la actividad forestal pase a un segundo plano.

El agotamiento y disminución de los recursos naturales en otras zonas genera la colonización de nuevos territorios para el aprovechamiento, situación que pone en riesgo la vegetación de la ciénaga La Grande. 


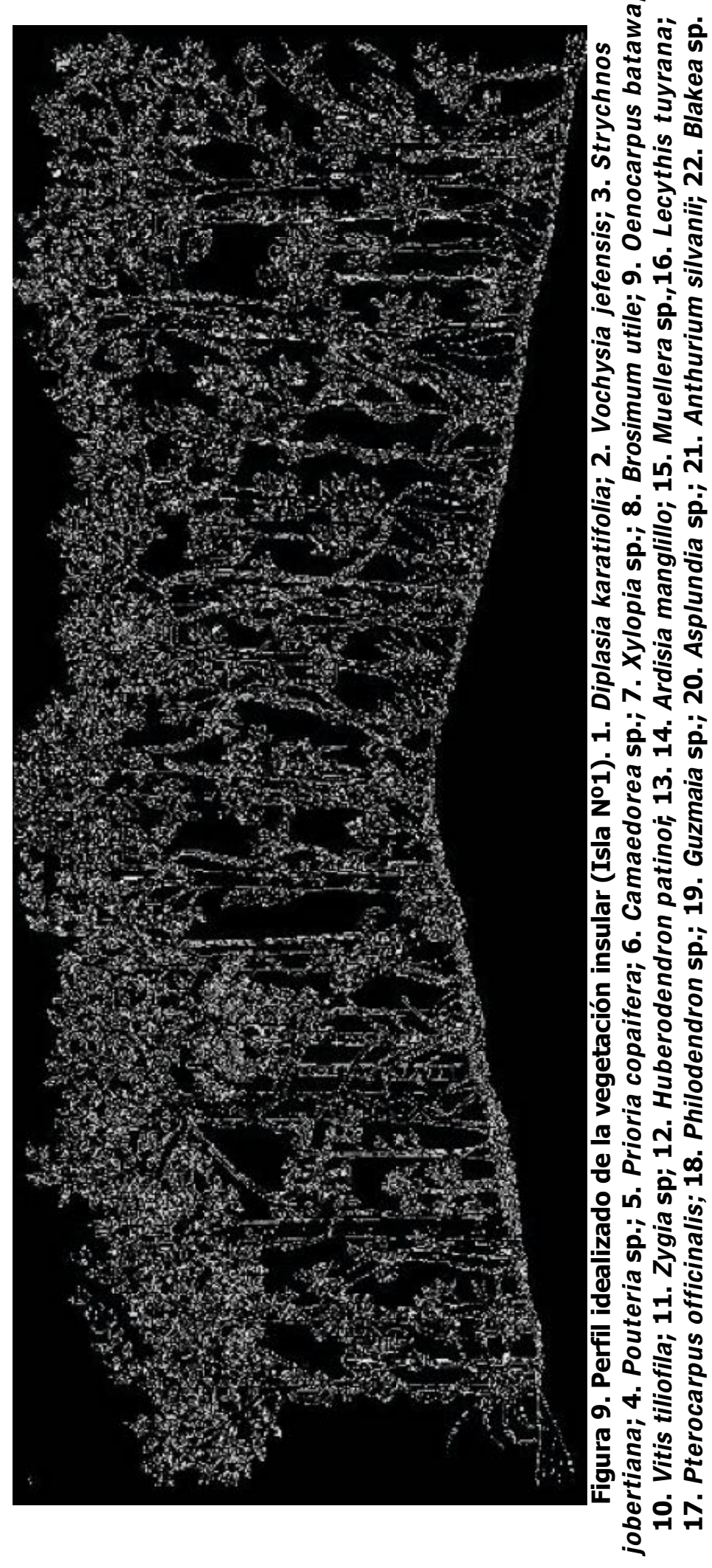

La explotación forestal que se está llevando a cabo en los alrededores de la ciénaga, se realiza sin ningún control lo que está trayendo como consecuencia la disminución de muchas especies incluyendo las que actualmente se encuentran reportadas en los libros rojos de Colombia.

La información obtenida a través de esta evaluación florística es la base para el conocimiento de la biodiversidad del ecosistema, además, sirve de sustento para tomar las decisiones que involucran su conservación.

\section{RECOMENDACIONES PARA SU CONSERVACIÓN}

Generar proyectos o programas alternativos que favorezcan a las comunidades, así como a la conservación del ambiente.

Fortalecer la investigación en estos ecosistemas que permita conocer su dinámica en varias épocas del año y generar información que describan el impacto de las actividades humanas en otros humedales de la región.

Establecer planes de manejo orientados al aprovechamiento de especies de importancia socioeconómica (forestales) reportadas en los libros rojos de Colombia y que al mismo tiempo permitan monitorearlas.

\section{RECOMENDACIONES COMO HERRAMIENTA EN EDUCACIÓN AMBIENTAL}

Desarrollar programas de educación ambiental y sensibilización a las comunidades asentadas en el municipio, con el fin de que se haga aprovechamiento de una forma racional del recurso forestal y así impedir el deterioro de los humedales.

Elaborar una guía didáctica que pueda ser consultada por docentes, estudiantes y pobladores en general resaltando el concepto de humedales, su importancia, las especies presentes y las que se encuentran bajo algún grado de amenaza.

\section{LITERATURA CITADA}

Andrade P. A., F. Navarrete Le B. 2004. Lineamientos para el enfoque ecosistémico a la gestión integral del recurso hídrico. Programa de las Naciones Unidas para el Medio Ambiente (PNUMA), Oficina Regional para América Latina y el Caribe. México D. F.; Red de Formación Ambiental.

Arévalo, R., J, Betancur. 2004. Diversidad de epifitas vasculares de cuatro bosques del sector sur-oriental de la serranía de Chiribiquete, Guayana colombiana. Caldasia 26 (2): 359-80. 


\section{Bioetnia Volumen 6 No 1 (enero-junio), 2009}

Betancur, J., A. Zuluaga, L. Clavijo, Z. Cordero-P, R. N. Salinas. 2007. Santa María pintada de flores. Serie de Guías de Campo del Instituto de Ciencias Naturales $\mathrm{N}^{\mathrm{o}}$ 1. Bogotá, D.C.: Instituto de Ciencias Naturales, Universidad Nacional de Colombia. $172 \mathrm{p}$

Bravo, C. L., J. Bravo-Ch. 2002. Refugio Nacional de Vida Silvestre Limoncito (RNVSL). Restauración y manejo de bosques inundables de palma (Raphia taedigera) y cativo (Prioria copaifera). Informe final. Programa Humedales de Costa Rica: Uso y Conservación. p. 7-27.

Cárdenas, L. D. 2003. Inventario florístico en el cerro del Cuchillo, Tapón del Darién colombiano. Caldasia 25 (1): 101-17.

Cárdenas, L. D. N. R. Salinas (eds.). 2007. Libro rojo de plantas de Colombia. Volumen 4. Especies maderables amenazadas: Primera parte. Serie libros rojos de especies amenazadas de Colombia. Bogotá, D.C.: Instituto Amazónico de Investigaciones Científicas SINCHI, Ministerio de Ambiente, Vivienda y Desarrollo Territorial. 232 pp.

Castellanos- M. C. A. 2006. Los ecosistemas de humedales en Colombia. Manizales: Universidad de Caldas.

Castillo, C, L., Johston, G, R. 2002. Evaluación de los humedales de los deltas de los ríos San Juan, Baudó y ciénegas de Tumarado, Perancho, la Honda y la Rica Bajo Atrato departamento del Chocó. Calidris. Cali: Ministerio del Medio Ambiente; $40 \mathrm{p}$.

Duque M. V., Gómez J. A, Palacios N. 2003. Plan de manejo del humedal los Platillos en el Medio Atrato (Antioquia,Chocó). Rev Semillas. 19: 15-20.

Fondo de Compensación Ambiental (FCA); Corporación para el Desarrollo Sostenible del Urabá (CORPOURABA); Corporación Autónoma Regio- nal para el Desarrollo Sostenible del Chocó (CODECHOCO). 2006. Plan de manejo integrado de los humedales del medio y bajo Atrato. URL disponible en: www.codechoco.gov.co

Instituto de Investigación de Recursos Biológicos Alexander Von Humboldt. 1998. Hacia la conservación de los humedales de Colombia: Biosintesis. 9: 4.

Moreno-Bejarano, L. M, R. Álvarez-León. 2003. Fauna asociada a los manglares y otros humedales en el delta-estuario del río Magdalena, Colombia. Rev Acad Colomb Cienc. 27 (105): 517-34.

Ramos P. Y. A. 2002. Evaluación de los humedales de los deltas de los ríos San Juan y Baudó y Ciénagas de Tumarado, Perancho, la Honda y la Rica, bajo Atrato, departamento del Chocó. Caracterización botánica y zoológica. Quibdó: Instituto de Investigaciones del Pacifico (IIAP), Ministerio del Medio Ambiente de Colombia, WWF-Programa Colombia

Rangel, J. O., Lowwi-C. P. D., Aguilar, M. 1997. Distribución de los tipos de vegetación en las regiones naturales de Colombia. En: Rangel, J. O., Lowwi-C. P. D., Aguilar, M. Colombia Diversidad Biótica II. Bogotá, D.C.; Universidad Nacional de Colombia; p. 383-402.

Rangel-Ch, O. 2004. Amenazas a la biota y a los ecosistemas del Chocó biogeográfico. En: Colombia Diversidad Biótica IV. El Chocó Biogeográfico/Costa Pacífica. Quibdó: ACIA.

Salazar, P.N.A. 2000. Vegetación asociada al medio Atrato.En: Plan de Manejo Ambiental del Territorio Colectivo de la ACIA. Quibdó: ACIA. 